\title{
ON THE DEGREES OF INDEX SETS. II
}

\author{
BY
}

C. E. M. YATES

In [8] we proved that the index-set corresponding to any recursively enumerable degree $a$ is of the highest isomorphism-type possible for sets belonging to $\Sigma_{3}(a)$. From the proof of this result we derived Sacks' theorem [4] that the recursively enumerable degrees are dense. In the present paper we classify three other indexsets associated with any given recursively enumerable degree $a$, namely the indexsets corresponding to the recursively enumerable sets which are respectively of degree $\leqq a, \geqq a$ and incomparable with $a$. We then use the indirect method of [8] to extend three theorems of Sacks ([3], [5]); these extensions were announced in [8]. Finally, amongst other results we infer from our classifications that certain enumerations are not recursively enumerable; for example, the recursively enumerable degrees (as distinct from sets) can not be recursively enumerated without repetitions.

We shall use most of the definitions employed in [8], but our notation will be slightly different and so we restate the more important definitions. One superficial but convenient change is that we shall do recursion-theory mainly on the positive integers rather than the nonnegative integers, since this leaves 0 free for various special purposes. Therefore, the terms "number" and "set" should be reinterpreted accordingly. We now assemble some of the definitions that we shall use. If $A$ is a set then we let $A(x)=1$ if $x \in A$ and $A(x)=2$ if $x \notin A$. For any number $e$ and set $A$ we define the partial function $\Theta_{e}^{A}$ by putting

$$
\Theta_{e}^{A}(x)=U\left(\min y T_{1}^{A}(e, x, y)\right) ;
$$

we follow the convention of setting $U(0)=0$. For each $e$ and $s$ we define

$$
x \in R_{e}^{s} \leftrightarrow(\exists y)_{y \leqq s} T_{1}(e, x, y) ;
$$

if $R_{e}=U_{s} R_{e}^{s}$ then $R_{1}, R_{2}, \ldots$, is an enumeration of all recursively enumerable sets. If $S$ is any recursively enumerable set then each number $e$ such that $R_{e}=S$ is called an index of $S$. We recall that an $n$-ary sequence $\left\{A_{i_{1} \ldots i_{n}}\right\}$ of recursively enumerable sets is called recursively enumerable if there is a recursive function $a\left(i_{1}, \ldots, i_{n}\right)$ such that $A_{i_{1} \ldots i_{n}}=R_{a\left(i_{1}, \ldots, i_{n}\right)}$ for all $i_{1}, \ldots, i_{n}$. A class of recursively enumerable sets is called recursively enumerable if it can be ordered into a recursively enumerable sequence.

The index-set $G(\mathscr{A})$ of a class $\mathscr{A}$ of sets is defined by

$$
e \in G(\mathscr{A}) \leftrightarrow R_{e} \in \mathscr{A} \text {. }
$$

In particular, the index-set $G(a)$ corresponding to a degree $a$ is defined by

$$
e \in G(a) \leftrightarrow R_{e} \text { is of degree } a .
$$

Received by the editors March 29, 1967 and, in revised form, August 16, 1967. 
The three other index-sets related to $a$ which we shall discuss in the present paper are defined by

$$
\begin{aligned}
& e \in G(\leqq a) \leftrightarrow R_{e} \text { is of degree } \leqq a, \\
& e \in G(\geqq a) \leftrightarrow R_{e} \text { is of degree } \geqq a, \\
& e \in G(\mid a) \leftrightarrow R_{e} \text { is of degree incomparable with } a .
\end{aligned}
$$

The various forms of the arithmetical hierarchy relative to a degree $\boldsymbol{a}$ are written as in [8]: $\Sigma_{n}(a), \Pi_{n}(a)$ are the classes of all sets expressible by a predicate form with $n$ alternating quantifiers the first of which is existential, universal respectively, where in each case the scope of the quantifiers is of degree $\leqq a$. We refer to $\Sigma_{n}(\mathbf{0})$ and $\Pi_{n}(0)$ simply as $\Sigma_{n}$ and $\Pi_{n}$. Our procedure for classifying an index-set $G(\mathscr{A})$ once again consists of finding a definition for $G(\mathscr{A})$ in some quantifier and then showing that $G(\mathscr{A})$ is of the highest isomorphism-type possible for sets in that form. The first part is trivial: in the cases we consider here, the obvious definitions suffice and we give these in the appropriate section. The second and difficult part reduces, as we explained in [8], to showing that if $S$ is any set in the same quantifier form as $G(\mathscr{A})$ then there is a recursively enumerable sequence $\left\{C_{k}\right\}$ of recursively enumerable sets such that for all $k$ :

$$
k \in S \leftrightarrow C_{k} \in \mathscr{A} .
$$

The layout of the paper is as follows. In $\S 1$ we obtain a representation for $\Sigma_{3}(a)$ which is slightly different from that used in [8]. In $\$ 2$ we show that if $a$ is a recursively enumerable degree $<\boldsymbol{0}^{(1)}$ then $G(\leqq a)$ is of the highest isomorphismtype possible for sets in $\Sigma_{3}(a)$. In $\S 3$ we use the techniques in the main section of [8] to show that if $\mathbf{0}<\boldsymbol{a} \leqq \boldsymbol{0}^{(1)}$ then $G(\geqq \boldsymbol{a})$ is of the highest isomorphsim-type possible for sets in $\Sigma_{4}$, and if $0<\boldsymbol{a}<\mathbf{0}^{(1)}$ then $G(\mid a)$ is of the highest isomorphism-type possible for sets in $\Pi_{4}$. In $\$ 4$ we prove:

(i) if $\boldsymbol{a}<\boldsymbol{0}^{(1)}$ and $\boldsymbol{a}_{1}<\boldsymbol{a}_{2}<\cdots$ is an infinite ascending recursively enumerable sequence of recursively enumerable degrees each $<\boldsymbol{a}$, then there is a recursively enumerable degree $\boldsymbol{c}$ such that $\boldsymbol{a}_{1}<\boldsymbol{a}_{2}<\cdots<\boldsymbol{c}$ and $\boldsymbol{a} \mid \boldsymbol{c}$.

(ii) if $\mathbf{0}<\boldsymbol{a}<\boldsymbol{0}^{(1)}$ then there is a recursively enumerable degree $\boldsymbol{c}$ such that $\boldsymbol{c}^{(1)}=\mathbf{0}^{(2)}$ and $\boldsymbol{a} \mid \boldsymbol{c}$. (We announced a stronger result in [8] but we have since been unable to carry through the proof of this.)

(iii) if $\mathbf{0}<\boldsymbol{a}<\mathbf{0}^{(1)}$ then there is a degree $\boldsymbol{c}$ which contains a maximal set and is such that $\boldsymbol{a} \mid \boldsymbol{c}$.

By a recent result of Martin [2], (iii) is in fact equivalent to (ii) so that we need only prove (ii). In a simple application of our method, we also prove in $\S 4$ that the maximal sets of degree $\mathbf{0}^{(1)}$ inhabit infinitely many isomorphism-types. Lastly, in $\S 5$ we derive some simple results about recursive enumerations.

1. Representation. In this section we devise a slightly stronger representation for sets in $\Sigma_{3}(a)$, where $\boldsymbol{a}$ is recursively enumerable, than that used in [8]. 
LEMMA 1. If $a$ is a recursively enumerable degree and $S \in \Sigma_{3}(a)$ then there is a recursively enumerable sequence $\left\{L_{k e x}\right\}$ having the following properties:

(1) for each $k$, the partial function $\lambda_{k}(e, x)=\max z\left(z \in L_{k e x}\right)$ is partial recursive in $a$.

(2) if $k \in S$ then there is an $e_{k}$ such that if $e \geqq e_{k}$ then $L_{k e x}$ is finite for all $x$.

(3) if $k \notin S$ then there is for each $e$ an $x_{e}$ such that $L_{k e x}$ is infinite for all $x \geqq x_{e}$.

Proof. Let $A$ be a fixed recursively enumerable set of degree $a$, and for each $s$ let $A^{s}$ be the finite subset of $A$ enumerated up to stage $s$ in some fixed recursive enumeration of $A$. Since $S \in \Sigma_{3}(a)$ there is a number $c$ such that for all $k$ :

$$
k \in s \leftrightarrow(\exists e)(\forall u)(\exists v) T_{3}^{A}(c, k, e, u, v) .
$$

Now define a recursively enumerable sequence $\left\{S_{k j i}\right\}$ by setting

$$
z \in S_{k e u} \leftrightarrow(\forall v)_{v<z}(\exists s)_{s \geqq z} \bar{T}_{3}^{A s}(c, k, e, u, v) .
$$

We first claim that the partial function

$$
z_{k}(e, u)=\max z \quad\left(z \in S_{k e u}\right)
$$

is partial recursive in $a$; for, it can be seen that

$$
\begin{aligned}
\max z\left(z \in S_{k e u}\right) & =\min z(\forall s)_{s \geqq z} T_{3}^{A^{s}}(c, k, e, u, z) \\
& =\min z\left[T_{3}^{A}(c, k, e, u, z) \& T_{3}^{A^{z}}(c, k, e, u, z)\right] .
\end{aligned}
$$

Our second claim is that for all $k$ :

$$
k \in S \leftrightarrow(\exists e)(\forall u) \quad\left(S_{k e u} \text { is finite }\right) .
$$

We leave the verification of this to the reader, since in any case a proof of this is contained in Lemma 4 of [8]. Now, for each $k, e$ and $x$ we define

$$
L_{k e x}=\bigcap_{f \leqq e} \bigcup_{u \leqq x} S_{k f u} \text {. }
$$

Clearly, $\left\{L_{k e x}\right\}$ is recursively enumerable, and if $\lambda_{k}(e, x)=\max z\left(z \in L_{k e x}\right)$ then it can be seen by examining the definition of $S_{l c f u}$ that $\lambda_{k}(e, x)=\min _{f \leqq e} \max _{u \leqq x} z_{k}(f, u)$ and so $\lambda_{k}$ is partial recursive in $a$. We leave the verification of (2) and (3) to the reader.

The representation that we used in [8] is easily obtained from Lemma 1 . For the sake of completeness we restate it here although we leave its derivation from Lemma 1 to the reader.

LEMMA 2. If $a$ is a recursively enumerable degree and $S \in \Sigma_{3}(a)$ then there is a recursively enumerable sequence $\left\{M_{k j}\right\}$ which is uniformly of degree $\leqq a$ and such that for all $k$ :

$$
\begin{aligned}
& k \in S \rightarrow(\exists e)\left(M_{k e} \text { is of degree a } \&(\forall j)_{j<e}\left(M_{k j} \text { is recursive }\right)\right), \\
& k \notin S \rightarrow(\forall e)\left(M_{k e} \text { is recursive }\right) .
\end{aligned}
$$

In the present paper our use of Lemma 2 will be confined to $\$ 3$ and $\$ 4$, where we employ it for a representation of sets in $\Sigma_{4}$-recall that $\Sigma_{4}=\Sigma_{3}\left(0^{(1)}\right)$. 
2. Classification of $G(\leqq a)$. If $a \geqq 0^{(1)}$ then $G(\leqq a)$ consists of all positive integers and so there is interest only in the case $a \geq 0^{(1)}$ and in particular $a<0^{(1)}$. In fact, we have only been able (and it only seems possible) to obtain a general classification in the principal case when $a$ is a recursively enumerable degree $<\mathbf{0}^{(1)}$.

We first need to obtain an upper bound for $G(\leqq a)$. This is very easy and was done in [8] but we may as well repeat it here. We observe:

$$
\begin{aligned}
e \in G(\leqq a) & \leftrightarrow R_{e} \text { is of degree } \leqq a \\
& \leftrightarrow(\exists f)(\forall x)\left(x \notin R_{e} \leftrightarrow(\exists y) T_{1}^{A}(f, x, y)\right),
\end{aligned}
$$

where $A$ is a fixed set of degree $a$. (The second equivalence holds because $R_{e}$ is recursive in $A$ if and only if $\bar{R}_{e}$ is recursively enumerable in $A$.) By eliminating $\leftrightarrow$ and bringing the predicate into normal form, it follows that $G(\leqq a) \in \Sigma_{3}(a)$. Notice that this is true for any degree $a$.

Now, we turn to showing that if $a$ is any recursively enumerable degree $<0^{(1)}$ then $G(\leqq a)$ is of the highest isomorphism-type available to sets in $\Sigma_{3}(a)$. One of the techniques that we use is essentially the easier half of Sacks' proof [4] that the recursively enumerable degrees are dense.

Before proving the theorem we need a few conventions about limits. If $f(x)$ is a function we say that $\lim _{x} f(x)=r$ if there is a number $x_{0}$ such that $f(x)=r$ for all $x \geqq x_{0}$ (in which case $\lim _{x} f(x)$ is finite), and that $\lim _{x} f(x)=\infty$ if for each $r$ there is a number $x_{r}$ such that $f(x)>r$ for all $x \geqq x_{r}$; otherwise, we say that $\lim _{x} f(x)$ does not exist.

THEOREM 1. If $a$ is a recursively enumerable degree $<0^{(1)}$ and $S \in \Sigma_{3}(a)$ then there is a recursively enumerable sequence $\left\{C_{k}\right\}$ of recursively enumerable sets such that : $k \in S \leftrightarrow C_{k}$ is of degree $\leqq a$.

Proof. Let $A$ be a fixed recursively enumerable set of degree $a$. Also, let $B$ be a fixed recursively enumerable set which is not recursive in $A$; such a set exists because $A$ is of degree $<0^{(1)}$. Let $A^{s}, B^{s}$ be the finite subsets of $A, B$ respectively, that have been enumerated up to stage $s$ in some stage-by-stage recursive enumeration of $A, B$. Let $\left\{L_{k e x}\right\}$ be the sequence related to $a$ and $S$ by Lemma 1 in $\S 1$, and let $L_{k e x}^{s^{\prime}}$ be the finite subset of $L_{k e x}$ enumerated up to stage $s$ in some uniform stage-bystage recursive enumeration of $\left\{L_{k e x}\right\}$. Finally, let $\lambda_{k}^{s}(e, x)$ be the largest element of $L_{\text {kex. }}^{s}$.

In preparation for the definition of $C_{k}$ we define

$$
\begin{array}{rlrl}
a(s, e, x) & =\min y_{y \leqq s} T_{1}^{A^{s}}(e, x, y) & & \text { if }(\exists y)_{y \leqq s} T_{1}^{A^{s}}(e, x, y), \\
& =0 & & \text { otherwise. } \\
z(s, e, x) & =a(s, e, x) & \text { if } a(s, e, x)>z(s-1, e, x), \\
& =z(s-1, e, x)+1 & & \text { if } a(s, e, x)=0, \\
& =z(s-1, e, x) & & \text { otherwise. }
\end{array}
$$


Clearly, if $\lim _{s} a(s, e, x)$ exists and is positive then $z_{e}(x)=\lim _{s} z(s, e, x)$ exists and is positive. Therefore, if $\Theta_{e}^{A}(x)$ is defined for all $x$ then $z_{e}(x)$ is defined for all $x$ and the function $z_{e}$ is recursive in $A$. On the other hand, if $\lim _{s} a(s, e, x)$ is $0, \infty$ or does not exist then $\lim _{s} z(s, e, x)=\infty$. Next, we define

$$
f_{k}(s, e)=\max \left\{f_{k}(s-1, e), \min x\left(U(a(s, e, x)) \neq C_{k}^{s-1}(x)\right)\right\} .
$$

Clearly, $\lim _{s} f_{k}(s, e)$ exists for each $e$, and if $\Theta_{e}^{A}(x)$ is defined for all $x$ then $\lim _{s} f_{k}(s, e)$ is finite if and only if $\Theta_{e}^{A}(x) \neq C_{k}(x)$ for some $x$. Finally we let $C_{k e}^{s}$ contain every element of $C_{k e}^{s-1}$ and every number $2^{e} \cdot 3^{y} \cdot 5^{z}$ such that

$\lambda_{k}^{s}(e, y) \neq \lambda_{k}^{s-1}(e, y) \&\left(\left(y \in B^{s} \& f_{k}(s, e) \geqq 2^{e} \cdot 3^{y} \cdot 5^{z}\right) \vee \max _{u \leqq y}\{z(s, e, u)\} \geqq z\right)$.

We define $C_{k}^{s}=\bigcup_{e} C_{k e}^{s}=\bigcup_{s} C_{k e}^{s}$ and $C_{k}=\bigcup_{s} C_{k}^{s}=\bigcup_{e} C_{k e}$, which completes the construction.

We begin the proof of the theorem with the following lemma.

LEMMA 3. Let $k$ and $e$ be fixed. If there is a $y_{e}$ such that $L_{k e y}$ is infinite for all $y \geqq y_{e}$ then there is an $x$ such that $\Theta_{e}^{A}(x)$ is undefined or $\Theta_{e}^{A}(x) \neq C_{k}(x)$.

Proof. Suppose, for reductio ad absurdum, that $\Theta_{e}^{A}(x)$ is defined and equal to $C_{k}(x)$ for all $x$. It follows that $\lim _{s} f_{k}(s, e)=\infty$, and that $z_{e}(x)$ is defined for all $x$. Hence, if $y \geqq y_{e}$ it can be seen that

$$
\begin{aligned}
& y \in B \rightarrow(\forall z)\left(2^{e} \cdot 3^{y} \cdot 5^{z} \in C_{k}\right), \\
& y \notin B \rightarrow(\forall z)_{z>\max _{u \unlhd y}\left(z_{e}(u)\right\}}\left(2^{3} \cdot 3^{y} \cdot 5^{z} \notin C_{k}\right),
\end{aligned}
$$

and so

$$
y \notin B \leftrightarrow(\exists z)\left(2^{e} \cdot 3^{y} \cdot 5^{z} \notin C_{k}\right) .
$$

Since $B$ is recursively enumerable, this implies that $B$ is recursive in $C_{k}$ and so as $B$ is not recursive in $A, C_{k}$ is not recursive in $A$.

If $k \notin S$ then by Lemma 1 of $\S 1$ there is for each $e$ a number $y_{e}$ such that $L_{k e y}$ is infinite for all $y \geqq y_{e}$, so that by Lemma 3 it follows that $\Theta_{e}^{A} \neq C_{k}$ for all $e$. Hence, if $k \notin S$ then $C_{k}$ is not recursive in $A$, which proves one side of the theorem.

If $k \in S$ then there is a number $e_{k}$ such that $\lambda_{k}(e, y)$ is defined for all $e \geqq e_{k}$ and all $y$. Moreover, since $\lambda_{k}$ is partial recursive in $A$ and since

$$
2^{e} \cdot 3^{y} \cdot 5^{z} \in C_{k e} \leftrightarrow(\exists r)_{r \leqq \min s\left(\lambda_{k}^{s}(e, y)=\lambda_{k}(e, y)\right)}\left(2^{e} \cdot 3^{y} \cdot 5^{z} \in C_{k e}^{r}\right)
$$

for each $e \geqq e_{k}$ and all $y$, it follows that $\bigcup_{e \geqq e_{k}} C_{k e}$ is recursive in $A$. It remains to prove that $\bigcup_{e<e_{k}} C_{k e}$ is recursive in $A$. Let $e$ be fixed and $<e_{k}$; then we simply wish to prove that $C_{k e}$ is recursive in $A$. Now, there is a $y_{e}$ such that for all $y \geqq y_{e}$ : $\lim _{s} \lambda_{k}^{s}(e, y)=\infty$ and so

$2^{e} \cdot 3^{y} \cdot 5^{z} \in C_{k e} \leftrightarrow\left(\left(y \in B \& \lim _{s} f_{k}(s, e) \geqq 2^{e} \cdot 3^{y} \cdot 5^{z}\right) \vee \max _{u \leqq y}\left\{\lim _{s} z(s, e, u)\right\} \geqq z\right)$. 
Case 1. $\Theta_{e}^{A}(x)$ is defined for all $x$. In this case, $\lim _{s} f_{k}(s, e)$ is finite by Lemma 3 and $z_{e}(y)$ is defined for all $y$, so that if $2^{e} \cdot 3^{y} \cdot 5^{z}>\lim _{s} f_{k}(s, e)$ then

$$
2^{e} \cdot 3^{y} \cdot 5^{z} \in C_{k e} \leftrightarrow z \leqq \max _{u \leqq y}\left\{z_{e}(u)\right\}
$$

It follows that $C_{k e}$ is recursive in $A$.

Case 2. $\Theta_{e}^{A}(x)$ is undefined for some $x$. In this case $\lim _{s} z\left(s, e, u_{e}\right)=\infty$ for some number $u_{e}$ and so $2^{e} \cdot 3^{y} \cdot 5^{z} \in C_{k e}$ for all $z$ if $y \geqq u_{e}$. It follows that $C_{k e}$ is the union of a finite number of finite or recursive sets and so is recursive. This completes the proof of Theorem 1 .

For reasons given in [8] we may immediately deduce:

COROllaRY 1.1. If $a$ is a recursively enumerable degree $<0^{(1)}$ then $G(\leqq a)$ is of the highest isomorphism-type possible for sets in $\Sigma_{3}(a)$.

This also yields the classification of $G(a)$ obtained in [8] for the case $a$ is a recursively enumerable degree $<\mathbf{0}^{(1)}$. For, if $A$ is a fixed recursively enumerable set. of degree $\boldsymbol{a}$ then there is a one-one recursive function $f$ such that

$$
2 x \in R_{f(e)} \leftrightarrow x \in A, \quad 2 x+1 \in R_{f(e)} \leftrightarrow x \in R_{e}
$$

for all $e$. Hence, $e \in G(\leqq a) \leftrightarrow f(e) \in G(a)$ for all $e$, so that $G(\leqq a)$ is one-one reducible to $G(a)$ which must then also be of the highest isomorphism-type possible for sets in $\Sigma_{3}(a)$. On the other hand, if $a=0^{(1)}$ then $G(\leqq a)$ is recursive and so the classification of $G(a)$ in this crucial case has to be dealt with as in [8].

Finally, we remark that the classification we have obtained will not extend to arbitrary $\boldsymbol{a}<\mathbf{0}^{(1)}$. For, P. F. Rowat has shown the existence of a minimal degree $a<0^{(1)}$ with $a^{(1)}=0^{(2)}$, so that $\Sigma_{3}(a)=\Sigma_{4}$ but $G(\leqq a)=G(0) \in \Sigma_{3}$ since $a$ is minimal and so cannot be recursively enumerable, which implies that $G(\leqq a)$ is not of the highest isomorphism-type possible for sets in $\Sigma_{3}(a)$.

3. Classification of $G(\geqq a)$ and $G(\mid a)$. If $a=0$ then $G(\geqq a)$ consists of all positive integers and $G(\mid a)$ is empty so that there is only real interest in the case $\mathbf{0}<\boldsymbol{a} \leqq \mathbf{0}^{(1)}$; in fact, $G(\mid a)$ is nontrivial if $a \mid 0^{(1)}$ but we shall not discuss this case here.

We first obtain upper bounds for $G(\geqq a)$ and $G(\mid a)$. Since $G(\mid a)$ is the complement of $G(\leqq a) \cup G(\geqq a)$, we shall obtain an upper bound for $G(\mid a)$ through obtaining one for $G(\geqq a)$. Let $a$ be $\leqq 0^{(1)}$ and let $A$ be a fixed set of degree $a$. We observe

$$
\begin{aligned}
e \in G(\geqq a) & \leftrightarrow R_{e} \text { is of degree } \geqq a \\
& \leftrightarrow(\exists f)(\forall x)\left(x \notin A \leftrightarrow(\exists y) T_{1}^{R_{e}}(f, x, y)\right) .
\end{aligned}
$$

Since $A$ is of degree $\leqq 0^{(1)}$ and $T_{1}^{R}(f, x, y)$ is of degree $\leqq 0^{(1)}$ as a predicate of $e, f, x, y$, it follows that $G(\geqq a) \in \Sigma_{3}\left(0^{(1)}\right)=\Sigma_{4}$. Now, because $G(\leqq a) \in \Sigma_{3}(a) \subseteq \Sigma_{4}$ we deduce that $G(\mid a) \in \Pi_{4}$. 
Our main task is to show that if $\mathbf{0}<\boldsymbol{a} \leqq \mathbf{0}^{(1)}$ then $G(\geqq a)$ is of the highest isomorphism-type possible for sets in $\Sigma_{4}$ and if $0<a<\boldsymbol{0}^{(1)}$ then $G(\mid a)$ is of the highest isomorphism-type possible for sets in $\Pi_{4}$. We prove a theorem that is slightly more general than necessary so that in the next section we may deduce, as an immediate corollary, the theorem on ascending sequences of degrees mentioned in the introduction; this involves only the most trivial alterations in the proof. Although this proof differs little in its general conception from that of the main theorem in [8], we give it in full detail since we have discovered what appears to be a much more elegant and comprehensible way of presenting it.

THEOREM 2. Let $\mathbf{0}<\boldsymbol{a} \leqq \mathbf{0}^{(1)}$ and let $\boldsymbol{a}_{1}<\boldsymbol{a}_{2}<\cdots$ be a recursively enumerable sequence of recursively enumerable degrees such that $a \ddagger a_{i}$ for all $i$. If $S \in \Sigma_{4}$ then there is a recursively enumerable sequence $\left\{C_{k}\right\}$ of recursively enumerable sets, each of which is of degree $\geqq a_{i}$ for all $i$, such that for each $k$ :

$k \in S \rightarrow C_{k}$ is of degree $\mathbf{0}^{(1)}$,

$k \notin S \rightarrow C_{k}$ is not of degree $\geqq a$.

Proof. Let $A$ be a fixed set of degree $\boldsymbol{a}$; since $\boldsymbol{a} \leqq \boldsymbol{0}^{(1)}$ there is a uniformly recursive sequence $\left\{A^{s}\right\}$ such that $\lim _{s} A^{s}(x)=A(x)$ for all $x$. Also, let $\left\{A_{i}\right\}$ be a recursively enumerable sequence of recursively enumerable sets, where $A_{i}$ is of degree $a_{i}$ for each $i$. Let $\left\{M_{k j}\right\}$ be the sequence shown to correspond to $S$ in Lemma 2 of $\S 1$; in other words $\left\{M_{k j}\right\}$ is recursively enumerable and such that for all $k$ :

$$
\begin{aligned}
& k \in S \rightarrow(\exists e)\left(M_{k e} \text { is of degree } 0^{(1)} \&(\forall j)_{j<e}\left(M_{k e} \text { is recursive }\right)\right), \\
& k \notin S<(\forall e)\left(M_{k e} \text { is recursive }\right) .
\end{aligned}
$$

We now define another recursively enumerable sequence $\left\{N_{k j}\right\}$ by setting

$$
\begin{aligned}
& N_{k j}=\left\{p_{j}^{x} \mid x \in A_{e}\right\} \quad \text { if } j=2 e, \\
& =\left\{p_{j}^{x} \mid x \in M_{k e}\right\} \quad \text { if } j=2 e+1 .
\end{aligned}
$$

(Here, $p_{j}$ is the $j$ th prime-number.) Since $a_{1}<a_{2}<\cdots, \bigcup_{i \leqq j} A_{i}$ is clearly of degree $a_{j}$ for all $j$. Therefore, $\left\{N_{k j}\right\}$ has the property that for all $k$ :

$$
\begin{aligned}
& k \in S \rightarrow(\exists e)\left(N_{k e} \text { is of degree } 0^{(1)} \& \bigcup_{j<e} N_{k j} \text { is not of degree } \geqq a\right), \\
& k \notin S \rightarrow(\forall e)\left(\bigcup_{j<e} N_{k j} \text { is not of degree } \geqq a\right) .
\end{aligned}
$$

Since $\left\{N_{k j}\right\}$ is recursively enumerable, we can let $N_{k j}^{S}$ be the finite subset of $N_{k j}$ enumerated up to stage $s$ in some fixed recursive enumeration of $\left\{N_{k j}\right\}$. For each $k$, we shall define $C_{k}$ to be $\bigcup_{j} C_{k j}$, where $C_{k j} \subseteq N_{k j}$ for all $j$. In order to give some feeling for the construction we mention now that we shall design it so that if $k \in S$ then $N_{k e}-C_{k e}$ is finite, where $e$ is the least number $2 j+1$ such that $M_{k j}$ is of degree $\mathbf{0}^{(1)}$, and if $k \notin S$ then $N_{k e}-C_{k e}$ is finite for all $e$. The former implies that if $k \in S$ then $C_{k}$ is of degree $\mathbf{0}^{(1)}$, so that $C_{k}$ is trivially of degree $\geqq a$ and $\geqq \boldsymbol{a}_{i}$ for all $i$. The latter implies that if $k \notin S$ then $C_{k}$ is of degree $\geqq a_{i}$ for all $i$, since for each $i$ there 
will in this case be a set $C_{k e}$ of degree $a_{i}$. So it can be seen now how we arrange that $C_{k}$ is of degree $\geqq a_{i}$ for all $i, k$.

We shall define $C_{k}$ stage by stage simultaneously with various functions that play a part in its definition, and $C_{k}^{s}$ will denote the finite set of numbers put into $C_{k}$ through our procedure up to stage $s$. We first define

$$
\begin{aligned}
\gamma_{k}(s, e, x) & =\min y_{y \leqq s} T_{1}^{C_{k}^{s-1}}(e, x, y) \quad \text { if }(\exists y)_{y \leqq s} T_{1}^{C_{k}^{s-1}}(e, y), \\
& =0 \text { otherwise. }
\end{aligned}
$$

Notice that if $\Theta_{e^{k}}^{C_{k}}(x)$ is defined for all $x$ then $\lim _{s} \gamma_{k}(s, e, x)$ exists and is positive, and $\Theta_{e}^{C_{k}}(x)=U\left(\lim _{s} \gamma_{k}(s, e, x)\right)$, for all $x$. On the other hand, if $\Theta_{e^{k}}^{C_{k}}(x)$ is undefined for some $x$ then either $\lim _{s} \gamma_{k}(s, e, x)=0, \lim _{s} \gamma_{k}(s, e, x)=\infty$ or $\lim _{s} \gamma_{k}(s, e, x)$ does not exist. Next we define

$$
\begin{aligned}
& f_{k}(s, e)=\max \left\{f_{k}(s-1, e), \min x\left(U\left(\gamma_{k}(s, e, x)\right) \neq A^{s}(x)\right)\right\}, \\
& g_{k}(s, e)=\min x\left(\gamma_{k}(s, e, x)=0 \vee \gamma_{k}(s, e, x) \neq \gamma_{k}(s-1, e, x)\right), \\
& h_{k}(s, e)=\min \left\{f_{k}(s, e), g_{k}(s, e)\right\}, \\
& d_{k}(s, e)=\max \left\{\gamma_{k}(s, e, x) \mid x<h_{k}(s, e)\right\} .
\end{aligned}
$$

Note that $\gamma_{k}(s, e, x)=0$ for all sufficiently large $x$, so that $U\left(\gamma_{k}(s, e, x)\right)=0 \neq A^{s}(x)$ for all sufficiently large $x$, and $f_{k}(s, e), g_{k}(s, e), h_{k}(s, e)$ and $d_{k}(s, e)$ are hence welldefined. It can be seen that $f_{k}$ is monotonic in $s$ for any fixed $e$, and also that if $\Theta_{e}^{C} e^{k}(x)$ is defined and equal to $A(x)$ for all $x$ then $\lim _{s} f_{k}(s, e)=\infty$. In addition, if $\Theta_{e}^{C} e^{k}(x)$ is defined for all $x$ then $\lim _{s} g_{k}(s, e)=\infty$. The most important definition in our construction is the following. If $z \in C_{k}^{s-1}$ then we set $\delta_{k}(s, e, z)=1$ and otherwise we define

$$
\begin{aligned}
\delta_{k}(s, e, z) & =0 \quad \text { if } z \leqq d_{k}(s, e) \&(\forall j)_{j<e}\left(\delta_{k}(s-1, j, z)=1\right) \\
& =1 \quad \text { if } z>d_{k}(s, e) \\
& =\delta_{k}(s-1, e, z) \quad \text { otherwise. }
\end{aligned}
$$

Finally, we put every element of $C_{k e}^{s-1}$ into $C_{k e}^{s}$ and otherwise put $z$ into $C_{k e}^{s}$ if $z \in N_{k e}^{s}$ and $\delta_{k}(s, j, z)=1$ for all $j \leqq e$. Then $C_{k}^{s}=\bigcup_{e} C_{k e}^{s}, C_{k e}=\bigcup_{s} C_{k e}^{s}$ and $C_{k}=$ $\bigcup_{s} C_{k}^{s}=\bigcup_{e} C_{k e}$. This completes the construction.

Before we turn to proving the theorem we need some important preliminaries. First, we observe that if $z \in N_{k e}$ then

$$
z \notin C_{k e} \leftrightarrow(\exists r)(\forall s)_{s \geqq r}(\exists j)_{j \leqq e}\left(\delta_{k}(s, j, z)=0\right) .
$$

Hence if we define

$$
z \in D_{k e} \leftrightarrow(\exists j)_{j \leqq e}(\exists r)(\forall s)_{s \geqq_{r}}\left(\delta_{k}(s, j, z)=0\right),
$$


then $N_{k e} \cap D_{k e} \subseteq N_{k e}-C_{k e}$. The following simple simple combinatorial lemma is crucial to our whole argument.

LeMMA 4. $N_{k e}-C_{k e}=N_{k e} \cap D_{k e}$, for all $k, e$.

Proof. We only have to prove that $N_{k e}-C_{k e} \subseteq D_{k e}$. Let $z \in N_{k e}-C_{k e}$ and suppose for reductio ad absurdum that $z \notin D_{k e}$. It follows that since $z \in N_{k e}-C_{k e}$ there is a largest number $f \leqq e$ such that $\delta_{k}(s, f, z)=0$ for infinitely many $s$, but that on the other hand since $z \notin D_{k e}$ there are then also infinitely many $s$ such that $\delta_{k}(s, f, z)=1$. Let $s^{*}$ be a stage such that if $s \geqq s^{*}$ then $\delta_{k}(s, j, z)=1$ for all $j$ such that $f<j \leqq e$; such a stage exists by choice of $f$. At each of the infinitely many stages $s>s^{*}$ for which $\delta_{k}(s, f, z)=0$ and $\delta_{k}(s-1, f, z)=1$ we must have $\delta_{k}(s-1, j, z)=1$ for all $j<f$ by definition of $\delta_{k}$. Since, if $s>s^{*}$ then $\delta_{k}(s-1, j, z)=1$ for all $j$ such that $f<j \leqq e$, it follows that $\delta_{k}(s-1, j, z)=1$ for all $j \leqq e$ at infinitely many stages $s>s^{*}$. Hence $z \notin N_{k e}-C_{k e}$, which proves the lemma by contradiction.

After this preliminary diversion, we turn to proving the theorem. We wish to prove that

$k \in S \rightarrow C_{k}$ is of degree $\mathbf{0}^{(1)}$,

$k \notin S \rightarrow A$ is not recursive in $C_{k}$.

This is an almost immediate consequence of the fact that for any fixed $k$ and for all $e$ :

$\mathrm{I}(e)$ : if $A$ is not recursive in $\bigcup_{j>e} N_{k j}$ then either $\Theta_{e}^{C} e^{k}(x)$ is undefined or $\Theta_{e}^{C}(x)$ $\neq A(x)$ for some $x$.

$\mathrm{II}(e):$ if $A$ is not recursive in $\bigcup_{j<e} N_{k j}$ then $D_{k e}$ is finite.

To see that it is a consequence we proceed as follows. If $k \in S$ then there is an $e$ such that $N_{k e}$ is of degree $\mathbf{0}^{(1)}$ and $A$ is not recursive in $\bigcup_{j<e} N_{k j}$. Hence, $D_{k e}$ is finite by $\mathrm{II}(e)$ and so $N_{k e}-C_{k e}$ is finite by Lemma 4 . It follows that $C_{k e}$ is of degree $\mathbf{0}^{(1)}$ and so $C_{k}$ is trivially of degree $\mathbf{0}^{(1)}$. On the other hand if $k \notin S$ then for all $e, A$ is not recursive in $\bigcup_{j<e} N_{k j}$; and so by $\mathrm{I}(e)$ it follows that $\Theta_{e}^{C} k(x)$ is either undefined or unequal to $A(x)$ for some $x$. Hence, if $k \notin S$ then $A$ is not recursive in $C_{k}$.

Our main task is, therefore, to prove with $k$ fixed that $\mathrm{I}(e)$ and $\mathrm{II}(e)$ hold for all $e$. We prove this by induction on $e$, first proving $\mathrm{I}(e)$ and then proving $\mathrm{I}(e) \rightarrow \mathrm{II}(e)$, in both cases assuming from our induction hypothesis that if $A$ is not recursive in $\bigcup_{j<e} N_{k j}$ then $D_{k j}$ is finite for all $j<\pi e$.

Before we can prove I $(e)$ we need a definition and two lemmas. We define a partial function $s_{e}$ by setting

$$
\begin{array}{r}
s_{e}(x)=\min s\left(h_{k}(s, e)>x \&(\forall y)_{y \leqq x}(\forall z)_{z \leqq \gamma_{k}(s, e, y)}\right. \\
\left(z \notin C _ { k } ^ { s - 1 } \rightarrow \left((\forall j)_{j<e}\left(z \in N_{k j} \rightarrow z \in D_{k j}\right)\right.\right. \\
\left.\left.\left.\quad \&\left(z \notin \bigcup_{j<e} D_{k j} \rightarrow \delta_{k}(s, e, z)=0\right)\right)\right)\right) .
\end{array}
$$

By hypothesis, $A$ is not recursive in the finite collection $\left\{N_{k j}\right)_{j<e}$. On the other hand, by hypothesis, $D_{k j}$ is finite for all $j<e$ and $s_{e}$ is partial recursive in the finite collection $\left\{N_{k j}\right\}_{j<e}$. Therefore, we can make the important observation that if $s_{e}(x)$ 
is defined for all $x$ then $A$ is not recursive in $s_{e}$. The two lemmas that we now prove show that $s_{e}(x)$ is defined if and only if there is an $r$ such that $h_{k}(s, e)>x$ for all $s \geqq r$.

LEMMA 5. If there is an $r$ such that $h_{k}(s, e)>x$ for all $s \geqq r$ then $s_{e}(x)$ is defined.

Proof. Suppose that $h_{k}(s, e)>x$ for all $s \geqq r$. Then $g_{k}(s, e)>x$ for all $s \geqq r$ and so $\gamma_{k}(s, e, y)=\gamma_{k}(r, e, y)>0$ for each $y \leqq x$ and all $s \geqq r$. Also, it then follows that $d_{k}(s, e) \geqq \max _{y \leqq x}\left\{\gamma_{k}(r, e, y)\right\}$ for all $s \geqq r$. We claim that if $z \notin C_{k}$ and $z \leqq \gamma_{k}(r, e, y)$ with $y \leqq x$ then there is a stage $p \geqq r$ such that for all $s \geqq p$ the following statements hold:

(1) $(\forall j)_{j<e}\left(z \in N_{k j} \rightarrow z \in D_{k j}\right)$,

(2) $z \notin \bigcup_{j<e} D_{k j} \rightarrow \delta_{k}(s, e, z)=0$.

The first of these statements is an immediate consequence of Lemma 4 and so we need only concern ourselves with (2). But if $z \notin \bigcup_{j<e} D_{k j}$ then there is a stage $p \geqq r$ such that $\delta_{k}(p-1, j, z)=1$ for all $j<e$. It follows that $\delta_{k}(p, e, z)=0$ and in fact $\delta_{k}(s, e, z)=0$ for all $s \geqq p$, since $z \notin C_{k}$ and $z \leqq d_{k}(s, e)$ for all $s \geqq r$. This completes the proof of our claim. As there are only finitely many $z \leqq \gamma_{k}(r, e, y)$ for $y \leqq x$, it is now easy to see that $s_{e}(x)$ is defined.

LeMma 6. If $s_{e}(x)$ is defined then $h_{k}(s, e)>x$ for all $s \geqq s_{e}(x)$.

Proof. If $s_{e}(x)$ is defined then $h_{k}\left(s_{e}(x), e\right)>x$ and so $f_{k}(s, e)>x$ for all $s \geqq s_{e}(x)$ by the monotonicity of $f_{k}$. Hence we have only to prove that $g_{k}(s, e)>x$ for all $s \geqq s_{e}(x)$. This amounts to proving that $\gamma_{k}(s, e, y)=\gamma_{k}\left(s_{e}(x), e, y\right)>0$ for all $y \leqq x$ and $s \geqq s_{e}(x)$. We prove this by induction on $s$. Suppose that $\gamma_{k}(s, e, y)=\gamma_{k}\left(s_{e}(x), e, y\right)$ for all $y \leqq x$ and all $s$ such that $s_{e}(x) \leqq s \leqq s^{\prime}$. We wish to prove that $\gamma_{k}\left(s^{\prime}+1, e, y^{\prime}\right)=$ $\gamma_{k}\left(s^{\prime}, e, y^{\prime}\right)$, where $y^{\prime} \leqq x$ is fixed in what follows, and since $\gamma_{k}\left(s^{\prime}, e, y^{\prime}\right)=\gamma_{k}\left(s_{e}(x), e, y\right)$ $>0$ this will be so if no number $z \leqq \gamma_{k}\left(s^{\prime}, e, y^{\prime}\right)$ belongs to $C_{k}^{s^{\prime}}-C_{k}^{s^{\prime}-1}$. Now, if $s_{e}(x) \leqq s \leqq s^{\prime}$ then $f_{k}(s, e) \geqq f_{k}\left(s_{e}(x), e\right) \geqq h_{k}\left(s_{e}(x), e\right)>x$, and $g_{k}(s, e)>x$ since $\gamma_{k}(s, e, y)$ $=\gamma_{k}(s-1, e, y)>0$ for all $y \leqq x$; for the case $s=s_{e}(x)$ this is so because $h_{k}\left(s_{e}(x), e\right)$ $>x$. Therefore, $h_{k}(s, e)>x$ and $d_{k}(s, e) \geqq \gamma_{k}(s, e, y)$ for each $y \leqq x$ and all $s$ such that $s_{e}(x) \leqq s \leqq s^{\prime}$. Now, suppose that $z \leqq \gamma_{k}\left(s^{\prime}, e, y^{\prime}\right)$ and that $z \notin C_{k}^{s^{\prime}-1}$ whence $z \notin C_{k}^{s^{(e x)-1}}$. First, it follows that if $z \in N_{k j}$ for some $j<e$ then $z \in D_{k j}$ and so $z \notin C_{k}$. Secondly, it follows that if $z \in \bigcup_{j \geqq e} N_{k j}$ then either $z \in \bigcup_{j<e} D_{k j}$ in which case $z \notin C_{k}$, or $z \notin \bigcup_{j<e} D_{k j}$ in which case $\delta_{k}\left(s_{e}(x), e, z\right)=0$. In the latter case, $\delta_{k}(s, e, z)=0$ for all $s$ such that $s_{e}(x) \leqq s \leqq s^{\prime}$, since $z \leqq d_{k}(s, e)$ for all $s$ such that $s_{e}(x) \leqq s \leqq s^{\prime}$, and so $z \notin C_{k}^{s^{\prime}}$. Hence, we have proved that

$$
\gamma_{k}\left(s^{\prime}+1, e, y^{\prime}\right)=\gamma_{k}\left(s^{\prime}, e, y^{\prime}\right)
$$

This completes the inductive proof that $\gamma_{k}(s, e, y)=\gamma_{k}\left(s_{e}(x), e, y\right)>0$ for each $y \leqq x$ and all $s \geqq s_{e}(x)$, which in turn implies that $h_{k}(s, e)>x$ for all $s \geqq s_{e}(x)$.

In order to prove $\mathrm{I}(e)$, we suppose for reductio ad absurdum that $\Theta_{e}^{C_{k}}(x)$ is defined and equal to $A(x)$ for all $x$ even though $A$ is not recursive in $\bigcup_{j<e} N_{k j}$. It follows 
that for each $x$ there is an $r$ such that $h_{k}(s, e)>x$ for all $s \geqq r$. Then, by Lemma 5 , $s_{e}(x)$ is defined for all $x$, and so for each $x$, by Lemma $6, h_{k}(s, e)>x$ for all $s \geqq s_{e}(x)$. Hence, $\gamma_{k}(s, e, x)=\gamma_{k}\left(s_{e}(x), e, x\right)>0$ for all $s \geqq s_{e}(x)$ and so $A(x)=\Theta_{e}^{C_{k}}(x)=$ $U\left(\gamma_{k}\left(s_{e}(x), e, x\right)\right)$. This implies that $A$ is recursive in $s_{e}$, which is the required contradiction.

To prove that $\mathrm{I}(e) \rightarrow \mathrm{II}(e)$, we proceed as follows. If $\Theta_{e^{k}}{ }^{k}(x)$ is undefined or unequal to $A(x)$ for some $x$, then there is a least $\bar{x}$ such that $h_{k}(s, e)=\bar{x}$ for infinitely many $s$. Also, there is a stage $\bar{s}$ such that $\gamma_{k}(s, e, x)=\gamma_{k}(\bar{s}, e, x)>0$ for each $x<\bar{x}$ and all $s \geqq \bar{s}$, since otherwise we would have $g_{k}(s, e)<\bar{x}$ and hence $h_{k}(s, e)<\bar{s}$ for infinitely many $s$. Now, $D_{k j}$ is finite for each $j<e$ by our induction hypothesis, and if $z \in D_{k e}-\bigcup_{j<e} D_{k j}$ then there is by Lemma 4 a number $s^{*}$ such that $z \leqq d_{k}(s, e)$ for all $s \geqq s^{*}$. Since there are infinitely many $s \geqq s^{*}$ such that

$$
d_{k}(s, e)=\max \left\{\gamma_{k}(\bar{s}, e, x) \mid x<\bar{x}\right\}
$$

we deduce that $D_{k e}$ is finite. This completes the proof that $\mathrm{I}(e)$ and $\mathrm{II}(e)$ hold for all $e$. We have already shown that this in turn implies that for all $k$ :

$k \in S \rightarrow C_{k}$ is of degree $\mathbf{0}^{(1)}$,

$k \notin S \rightarrow C_{k}$ is not of degree $\geqq a$.

Also, we have shown above that it can be easily deduced that $C_{k}$ is of degree $\geqq a_{i}$ for all $i, k$; in the case that $k \in S$ this is immediate and in the case that $k \notin S$ it depends simply on Lemma 4 and the fact that then $D_{k e}$ is finite for all $e$. Hence we have proved Theorem 2 .

COROLlaRY 2.1. If $0<a \leqq 0^{(1)}$ then $G(\geqq a)$ is of the highest isomorphism-type possible for sets in $\Sigma_{4}$.

In order to classify $G(\mid a)$ with $0<a<0^{(1)}$, we need Sacks' theorem [3] that if $\mathbf{0}<\boldsymbol{a}<\boldsymbol{0}^{(1)}$ then there is a recursively enumerable degree incomparable with $\boldsymbol{a}$. This theorem can in fact be deduced from Theorem 2, which is of some interest in itself, and in Theorem 3 of the next section we actually derive a stronger result from Theorem 2.

Corollary 2.2. If $0<a<0^{(1)}$ then $G(\mid a)$ is of the highest isomorphism-type possible for sets in $\Pi_{4}$.

Proof. Let $\boldsymbol{b}$ be a recursively enumerable degree incomparable with $\boldsymbol{a}$, and let $S \in \Pi_{4}$. By Theorem 2 there is a recursively enumerable sequence of recursively enumerable sets $\left\{C_{k}\right\}$ each of degree $\geqq b$ and such that for all $k$ :

$k \in S \rightarrow C_{k}$ is not of degree $\geqq a$,

$k \notin S \rightarrow C_{k}$ is of degree $\mathbf{0}^{(1)}$.

Therefore, if $k \notin S$ then $C_{k}$ is not of degree incomparable with $a$, but if $k \in S$ then $C_{k}$ is not of degree $\geqq a$, and $C_{k}$ is not of degree $\leqq a$ since this would imply that $\boldsymbol{b} \leqq a$. Hence, we have proved that for all $k$ :

$k \in S \leftrightarrow C_{k}$ is of degree incomparable with $a$. 
It follows that $G(\mid a)$ is of the highest isomorphism-type possible for sets in $\Pi_{4}$. In the next section we shall turn from classifying index-sets to deriving theorems on recursively enumerable degrees by the indirect method we used in [8].

4. Extending some theorems of Sacks. It was proved by Sacks [3] that $\mathbf{0}^{(1)}$ is not a minimal upper bound for an infinite ascending sequence of uniformly recursively enumerable degrees; this theorem can be seen to be an immediate corollary of our Theorem 2. Sacks in fact proved that if $\boldsymbol{a}_{1}<\boldsymbol{a}_{2}<\cdots$ is an infinite ascending sequence of uniformly recursively enumerable degrees each $<a$, where $\boldsymbol{a} \leqq \boldsymbol{0}^{(1)}$, then there is a recursively enumerable degree $\boldsymbol{c}$ such that $\boldsymbol{a}_{1}<\boldsymbol{a}_{2}<\cdots<\boldsymbol{c}$ and $\boldsymbol{a} \leq \boldsymbol{c}$; this is again an immediate corollary of our Theorem 2, but in fact for $\boldsymbol{0}<\boldsymbol{a}<\boldsymbol{0}^{(1)}$ we can derive the much stronger theorem that follows. We phrase it in slightly more precise language so as to avoid any confusion.

THEOREM 3. If $0<\boldsymbol{a}<\mathbf{0}^{(1)}$ and $\boldsymbol{a}_{1}<\boldsymbol{a}_{2}<\cdots$ is an infinite recursively enumerable sequence of recursively enumerable degrees each $<a$, then there is a recursively enumerable degree $c$ such that $a_{1}<a_{2}<\cdots<c$ and $a \mid c$.

Proof. Since $G(\leqq a) \in \Sigma_{4}$ it follows from Theorem 2 that there is a recursively enumerable sequence $\left\{\boldsymbol{C}_{k}\right\}$ of recursively enumerable sets each of degree $\geqq \boldsymbol{a}_{i}$ for all $i$ and such that for all $k:$ if $R_{k}$ is of degree $\leqq a$ then $C_{k}$ is of degree $\mathbf{0}^{(1)}$ and if $R_{k}$ is not of degree $\leqq a$ then $C_{k}$ is not of degree $\geqq a$. By the recursion theorem, there is a $k_{0}$ such that $R_{k_{0}}=C_{k_{0}}$ and it is clear that the recursively enumerable set $R_{k_{0}}=C_{k_{0}}$ is of degree incomparable with $a$ but $\geqq a_{i}$ for all $i$. This completes the proof of Theorem 3.

Our next result is a partial extension of Sacks' main theorem [3] on the jump operator: if $\mathbf{0}<\boldsymbol{a} \leqq \boldsymbol{0}^{(1)}$ and $\boldsymbol{b}$ is a degree $\geqq \boldsymbol{0}^{(1)}$ which is recursively enumerable in $\mathbf{0}^{(1)}$, then there is a recursively enumerable degree $\boldsymbol{c}$ such that $\boldsymbol{c}^{(1)}=\boldsymbol{b}$ and $\boldsymbol{a}$ 主c . We announced incorrectly in [8] that if $\mathbf{0}<\boldsymbol{a}<\boldsymbol{0}^{(1)}$ then we could replace $\boldsymbol{a} \pm \boldsymbol{c}$ in Sacks' theorem by $a \mid c$. In fact we can only do this in the important particular case $\boldsymbol{b}=\mathbf{0}^{(2)}$, and we conjecture that it is false for some $\boldsymbol{b} \geqq \boldsymbol{0}^{(1)}$ and recursively enumerable in $\mathbf{0}^{(1)}$.

THEOREM 4. Suppose that $\mathbf{0}<\boldsymbol{a}<\mathbf{0}^{(1)}$. If $S \in \Sigma_{4}$ then there is a recursively enumerable set such that for all $k$ :

$k \in S \rightarrow C_{k}$ is of degree $\mathbf{0}^{(1)}$,

$k \notin S \rightarrow C_{k}$ is not of degree $\geqq a \& C_{k}^{(1)}$ is of degree $\mathbf{0}^{(2)}$.

Proof. Let $A$ be a fixed set of degree $\boldsymbol{a}$; since $\boldsymbol{a}<\boldsymbol{0}^{(1)}$ there is a uniformly recursive sequence $\left\{A^{s}\right\}$ such that $\lim _{s} A^{s}(x)$ exists and is equal to $A(x)$ for all $x$. Also, let $B$ be a fixed set of degree $\mathbf{0}^{(2)}$; since $\mathbf{0}^{(2)}$ is recursively enumerable in $\mathbf{0}^{(1)}$, there is a recursively enumerable sequence $\left\{B_{e}\right\}$ such that for all $e$ :

$e \in B \rightarrow B_{e}$ is finite,

$e \notin B \rightarrow(\forall z)\left(z \in B_{e}\right)$. 
Let $\left\{M_{k j}\right\}$ be the recursively enumerable sequence shown to correspond to $S$ in Lemma 2 of $\S 1$. We define another recursively enumerable sequence $\left\{N_{k j}\right\}$ by setting

$$
\begin{aligned}
& N_{k j}=\left\{p_{j}^{x} \mid x \in B_{e}\right\} \quad \text { if } j=2 e, \\
& =\left\{p_{j}^{x} \mid x \in M_{k e}\right\} \quad \text { if } j=2 e+1 \text {. }
\end{aligned}
$$

Clearly, for all $k$ :

$k \in S \rightarrow(\exists e)\left(N_{k e}\right.$ is of degree $\mathbf{0}^{(1)} \&(\forall j)_{j<e}\left(N_{k j}\right.$ is recursive $\left.)\right)$,

$k \notin S \rightarrow(\forall e)\left(N_{k e}\right.$ is recursive $)$.

Since $\left\{N_{k j}\right\}$ is recursively enumerable, we may let $N_{k j}^{s}$ be the finite subset of $N_{k j}$ enumerated up to stage $s$ in some fixed recursive enumeration of $\left\{N_{k j}\right\}$. We shall define $C_{k}$ to be $\bigcup_{j} C_{k j}$, where $C_{k} \subseteq N_{k j}$ for all $j$. Once again we shall arrange that if $k \in S$ then $N_{k e}-C_{k e}$ is finite, where $\bar{e}$ is the least number $2 j+1$ such that $M_{k j}$ is of degree $\mathbf{0}^{(1)}$, and that if $k \notin S$ then $N_{k e}-C_{k e}$ is finite for all $e$. The former implies that if $k \in S$ then $C_{k}$ is of degree $0^{(1)}$. The latter implies that if $k \notin S$ then for all $e$ :

$e \in B \leftrightarrow(\exists u)(\forall v)_{u>v}\left(p_{2 e}^{v} \notin C_{k}\right)$,

$e \notin B \leftrightarrow(\exists u)(\forall v)_{v>u}\left(p_{2 e}^{v} \in C_{k}\right)$.

Hence, if $k \notin S$ then $B$ is recursive in $C_{k}^{(1)}$ and so $C_{k}^{(1)}$ is of degree $0^{(2)}$. The rest of the theorem goes through as in Theorem 2.

COROLlARY 4.1. If $0<a<0^{(1)}$ then there is a recursively enumerable degree c such that $a \mid c$ and $c^{(1)}=0^{(2)}$.

Proof. Let $\left\{C_{k}\right\}$ be the sequence shown to exist in Theorem 4 corresponding to $G$ (ฏa) since $G(\leqq a) \in \Sigma_{4}$. By the recursion theorem there is a $k_{0}$ such that $R_{k_{0}}=C_{k_{0}}$ is of degree incomparable with $a$ and such that $C_{k_{0}}^{(1)}$ is of degree $\mathbf{0}^{(2)}$.

We recall that a recursively enumerable set $M$ is maximal if every recursively enumerable extension of $M$ either has a finite complement or differs from $M$ by a finite set. Sacks [5] proved that if $\mathbf{0}<\boldsymbol{a} \leqq \mathbf{0}^{(1)}$ then there is a degree $\boldsymbol{c}$, such that $\boldsymbol{a} \leq \boldsymbol{c}$ and $c$ contains a maximal set; Martin [2] has since proved that a recursively enumerable degree $c$ is the degree of a maximal set if and only if $c^{(1)}=0^{(2)}$ and so this result of Sacks is in fact an immediate corollary of Sacks' own work [3] on the jump-operator. Martin's theorem also enables us to state

COROLlaRY 4.2. If $\mathbf{0}<\boldsymbol{a}<\boldsymbol{0}^{(1)}$ then there is a recursively enumerable degree c such that $a \mid c$ is the degree of a maximal set.

This extends Sacks' theorem on maximal sets. Another result which has been consolidated by Martin's theorem is our theorem [7] that there is a maximal set of degree $0^{(1)}$. A simple application of our present methods enables us to state a more general theorem with which to conclude this section.

THEOREM 5. The maximal sets of degree $\mathbf{0}^{(1)}$ lie in an infinity of incomparable many-one degrees. 
Proof. We first need the following lemma due to Paul Young which is of some interest in its own right. Since it is unpublished, we give our own proof here.

LEMMA 7. Any two maximal sets are either many-one incomparable or of the same many-one degree.

Proof. Suppose that $A$ and $B$ are two maximal sets, $A$ is many-one reducible to $B$ and $f$ is a recursive function such that for all $x: x \in A \leftrightarrow f(x) \in B$. Let $F$ be the range of $f$. Then $B \cup f(\bar{A})=B \cup F$ and so is a recursively enumerable superset of $B$. As $B$ is maximal, $f(\bar{A})$ must be either finite or differ from $\bar{B}$ by a finite set. But $\bar{A}$ is many-one reducible to $f(\bar{A})$ and so $f(\bar{A})$ cannot be finite since this would imply that $\bar{A}$ is recursive. We conclude that $f(\bar{A})$ differs from $\bar{B}$ by a finite set $Q$. Let $p$ and $q$ be arbitrary elements of $A$ and $\bar{A}$ respectively, and let $b$ be a recursive function ranging over $B$. We set

$$
\begin{aligned}
g(x) & =p \quad \text { if }(\exists y)\left(b(y)=x \&(\forall z)_{z \leqq y}(f(z) \neq x)\right), \\
& =q \quad \text { if } x \in Q, \\
& =\min y\left(f(y)=x \&(\forall z)_{z<y}(b(z) \neq x)\right) \text { otherwise. }
\end{aligned}
$$

We leave the reader to satisfy himself that $g$ is recursive and that for all $x: x \in B \leftrightarrow g(x) \in A$. This completes the proof of the lemma. Notice that in the proof of the lemma we did not need the maximality of $A$ but simply that $A$ is not recursive; so the maximal sets inhabit minimal many-one degrees.

It remains to prove that the maximal sets of degree $\mathbf{0}^{(1)}$ lie in an infinity of manyone degrees. Suppose that, on the contrary, they lie in only finitely many many-one degrees $\mathscr{A}_{1}, \mathscr{A}_{2}, \ldots, \mathscr{A}_{n}$. It follows easily from Lemma 1 of [8] that

$$
G\left(\mathscr{A}_{1} \cup \ldots \cup \mathscr{A}_{n}\right) \in \Sigma_{3}
$$

in other words there is a recursive predicate $\Gamma$ such that for all $k$ :

$$
R_{k} \in \mathscr{A}_{1} \cup \cdots \cup \mathscr{A}_{n} \leftrightarrow(\exists e)(\forall y)(\exists z) \Gamma(k, e, y, z) .
$$

We now first describe a recursive enumeration of recursively enumerable sets $C_{1}, C_{2}, \ldots$, such that

$$
\begin{aligned}
& R_{k} \in \mathscr{A}_{1} \cup \ldots \cup \mathscr{A}_{n} \rightarrow C_{k} \text { is recursive, } \\
& R_{k} \notin \mathscr{A}_{1} \cup \ldots \cup \mathscr{A}_{n} \rightarrow C_{k} \text { is maximal and of degree } \mathbf{0}^{(1)} .
\end{aligned}
$$

We then use the recursion theorem to obtain a contradiction.

Since the sets $C_{1}, C_{2}, \ldots$, are obtained by incorporating a very simple procedure into the construction of a maximal set of degree $0^{(1)}$, we shall not go into details but merely describe the procedure assuming that the reader is familiar with [7]. The basic idea of [7] is to define a maximal set $M$ such that $\bar{M}$ dominates every partial recursive function, for it must then be of degree $0^{(1)}$ by an observation due to Tennenbaum. This is achieved by starting with a recursive function $t$ which is assumed only to be such that $t(e, s) \leqq t(e, s+1)$ and $t(e, s)<t(e+1, s)$ for all $e$ and 
$s$, and $t(e)=\lim _{s} t(e, s)$ exists for all $e$. Next, $M$ is constructed so that if $u_{1}, u_{2}, \ldots$, are elements of $\bar{M}$ in increasing order then $u_{e}>t(e)$ for all $e$. Finally, it is shown that if $t$ is suitably chosen then $\bar{M}$ dominates every partial recursive function. With these observations in mind we shall now show how to produce the required sets $C_{1}, C_{2}, \ldots$ First, we need to define

$$
d_{k}(s, e)=\max v(\exists f)_{f \leqq e}(\forall y)_{y \leqq v}(\exists z)_{z \leqq s} \Gamma(k, f, y, z)
$$

for each $k, e$ and $s$. Clearly, if $R_{k} \in \mathscr{A}_{1} \cup \cdots \cup \mathscr{A}_{n}$ then there is a least number $e_{k}$ such that $d_{k}(s, e)$ takes on infinitely many values as $s$ increases, for each $e \geqq e_{k}$. On the other hand, if $R_{k} \notin \mathscr{A}_{1} \cup \cdots \cup \mathscr{A}_{n}$ then $\lim _{s} d_{k}(s, e)$ exists and is finite for all $e$. Let $k$ now be fixed but arbitrary. We define $t_{k}(s, e)=\max \left\{t(e, s), d_{k}(s, e)\right\}$ for for all $e$ and $s$, and proceed to define $C_{k}$ exactly as $M$ is defined in [7] except that we use $t_{k}$ instead of $t$. If $R_{k} \notin \mathscr{A}_{1} \cup \cdots \cup \mathscr{A}_{n}$ then $t_{k}(e)=\lim _{s} t_{k}(s, e)$ exists and is finite for all $e$, and since it has all the properties of $t$ mentioned above (for, if the sequence $t_{1}, t_{2}, \ldots$, dominates every recursive function then so does the sequence $\left.t_{k 1}, t_{k 2}, \ldots\right)$ the corresponding set $C_{k}$ is a maximal set of degree $0^{(1)}$. On the other hand, if $R_{k} \in \mathscr{A}_{1} \cup \cdots \cup \mathscr{A}_{n}$ then there is a number $e_{k}$ such that $t_{k}(s, e)$ takes on infinitely many values for each $e \geqq e_{k}$, whence it will be seen by inspecting the construction in [7] that the corresponding set $\bar{C}_{k}$ is finite.

Since the construction that we have outlined is uniformly effective, there is a recursive function $\gamma$ such that $C_{k}=R_{\gamma(k)}$ for all $k$. By the recursion theorem there is a number $k^{*}$ such that $R_{k^{*}}=R_{\gamma\left(k^{*}\right)}$ and it is clear that $R_{k^{*}} \notin \mathscr{A}_{1} \cup \cdots \cup \mathscr{A}_{n}$ although $R_{k^{*}}$ is a maximal set of degree $0^{(1)}$. This completes the proof of the theorem.

It is possible to prove Theorem 5 by a difficult direct construction. This was done by Martin (unpublished) and it was on being told of his proof that we discovered the much simpler proof above.

Finally, we announced in [8] that the index-sets corresponding to the maximal sets and the hyperhypersimple sets are both of the highest isomorphism-type possible for sets in $\Pi_{4}$. We leave the proof of these results to the interested reader but remark that they have also been obtained independently by A. H. Lachlan and R. W. Robinson.

5. Recursive enumeration. In this final section we shall use two of the index-set classifications that we have obtained here and in [8] to prove the nonexistence of recursive enumerations satisfying certain conditions.

First, we use the classification of $G\left(\mathbf{0}^{(1)}\right)$ that was obtained in [8] to show that the recursively enumerable degrees can not be recursively enumerated without repetitions. (We recall that Friedberg [1] has proved that the recursively enumerable sets can be recursively enumerated without repetitions.) This is an immediate consequence of the following rather stronger theorem.

THEOREM 6. If $\left\{A_{j}\right\}$ is a sequence of recursively enumerable sets such that to each recursively enumerable degree a there corresponds exactly one number jor which $A_{j}$ is of degree a, then $\left\{A_{j}\right\}$ is not uniformly of degree $\leqq 0^{(1)}$. 
Proof. Suppose for reductio ad absurdum that $\left\{A_{j}\right\}$ is uniformly of degree $\leqq 0^{(1)}$. Let $\left\{A_{j}^{*}\right\}$ be the sequence obtained from $\left\{A_{j}\right\}$ by omitting the set $A_{j}$ that represents $\mathbf{0}^{(1)}$; clearly, the sequence $\left\{A_{j}^{*}\right\}$ remains uniformly of degree $\leqq 0^{(1)}$. Now, for each $e$, $R_{e}$ is of degree $<\boldsymbol{0}^{(1)}$ if and only if $R_{e}$ is recursive in $A_{j}^{*}$ for some $j$. Therefore, it follows that

$$
\begin{aligned}
G\left(0^{(1)}\right) & =\left\{e \mid(\forall j)\left(R_{e} \text { is not recursive in } A_{j}^{*}\right)\right\} \\
& =\left\{e \mid(\forall j)(\forall f)(\exists x)\left(x \in R_{e} \leftrightarrow(\exists y) T_{1} A_{j}^{*}(f, x, y)\right)\right\},
\end{aligned}
$$

since $R_{e}$ is recursive in $A_{j}^{*}$ if and only if $\bar{R}_{e}$ is recursively enumerable in $A_{j}^{*}$. Now, because $\left\{A_{j}^{*}\right\}$ is uniformly of degree $\leqq 0^{(1)}$, it follows by removing $\leftrightarrow$ and standard contraction that $G\left(\mathbf{0}^{(1)}\right) \in \Pi_{4}$. This contradicts our theorem [8] that $G\left(0^{(1)}\right)$ is of the highest isomorphism-type possible for sets in $\Sigma_{4}$ and so does not belong to $\Pi_{4}$.

THEOREM 7. There is no recursively enumerable sequence $\left\{A_{j}\right\}$ of recursively enumerable sets such that to each recursively enumerable degree a there corresponds exactly one number $j$ for which $A_{j}$ is of degree a.

Proof. Every recursively enumerable sequence of recursively enumerable sets is uniformly of degree $\leqq \mathbf{0}^{(1)}$.

Now we turn to our second result. Let $\boldsymbol{a}$ be any recursively enumerable degree. We shall obtain a necessary and sufficient condition for the recursively enumerable sets of degree $\leqq a$ to be uniformly recursively enumerable or uniformly of degree $\leqq 0^{(1)}$. As a consequence of this result we obtain a number of recursively enumerable degrees $\boldsymbol{a}$ for which the recursively enumerable sets of degree $\leqq a$ are not uniformly of degree $\leqq \mathbf{0}^{(1)}$, and so in particular not uniformly recursively enumerable. We need the following preliminary theorem which is of some interest in its own right.

THEOREM 8. If $\mathscr{A}$ is a class of recursively enumerable sets which contains all finite sets then the following are equivalent:

(i) $\mathscr{A}$ is recursively enumerable,

(ii) $\mathscr{A}$ is uniformly of degree $\leqq \mathbf{0}^{(1)}$,

(iii) $G(\mathscr{A}) \in \Sigma_{3}$.

Proof. (i) $\rightarrow$ (ii) since every recursively enumerable class of recursively enumerable sets is uniformly of degree $\leqq 0^{(1)}$. In order to prove that (ii) $\rightarrow$ (iii), let $\mathscr{A}$ be uniformly of degree $\leqq 0^{(1)}$. It follows that there is a function $a(j, x)$ of degree $\leqq 0^{(1)}$ such that every element of $\mathscr{A}$ is represented by $a(j, x)$ for some $j$. In other words we can write

$$
e \in G(\mathscr{A}) \leftrightarrow(\exists j)(\forall x)\left(R_{e}(x)=a(j, x)\right) .
$$

It easily follows that $G(\mathscr{A}) \in \Sigma_{3}$ since $\Sigma_{3}=\Sigma_{2}\left(0^{(1)}\right)$. Lastly, in order to prove that 
(iii) $\rightarrow$ (i) we suppose that $G(\mathscr{A}) \in \Sigma_{3}$. Let $\Gamma$ be a recursive predicate such that for all $e$ :

$$
e \in G(\mathscr{A}) \leftrightarrow(\exists j)(\forall y)(\exists z) \Gamma(e, j, y, z) .
$$

We define a recursively enumerable sequence $\left\{A_{k}\right\}$ by setting

$$
x \in A_{k} \leftrightarrow x \in R_{(k)_{1}} \&(\forall y)_{y \leqq x}(\exists z) \Gamma\left((k)_{1},(k)_{2}, y, z\right) .
$$

If $A_{k}$ is finite then $A_{k} \in \mathscr{A}$ by hypothesis. If $A_{k}$ is infinite then $(k)_{1} \in G(\mathscr{A})$ and $A_{k}=R_{(k)_{1}}$ so that $A_{k} \in \mathscr{A}$. On the other hand, if $R_{e} \in \mathscr{A}$ then $(\forall y)(\exists z) \Gamma(e, f, y, z)$ for some least number $f$; let $k=2^{e} \cdot 3^{f}$. It follows that $A_{k}=R_{e}$ and so the elements of $\left\{A_{k}\right\}$ exactly constitute $\mathscr{A}$. This completes the proof of the theorem.

Since $G(\mathbf{0}) \in \Sigma_{3}$ and every finite set is of degree 0 it follows immediately from Theorem 8 that the recursive sets are uniformly recursively enumerable; this was first proved by Suzuki [6]. The more general theorem we have proved, however, provides recursive enumerations for a number of less obvious classes: for example, for the class of recursively enumerable sets of degree $\leqq a$ whenever $a^{(2)}=0^{(2)}$, since then $\Sigma_{3}(a)=\Sigma_{3}$ and so $G(\leqq a) \in \Sigma_{3}$. In fact, we have a necessary and sufficient condition for the existence of such recursive enumerations in the following theorem.

THEOREM 9. If $\boldsymbol{a}$ is any recursively enumerable degree $<0^{(1)}$ then the following are equivalent:

(i) the class of recursively enumerable sets of degree $\leqq a$ is uniformly recursively enumerable,

(ii) the class of recursively enumerable sets of degree $\leqq a$ is uniformly of degree $\leqq 0^{(1)}$,

(iii) $a^{(2)}=0^{(2)}$.

Proof. (i) and (ii) are equivalent by the preceding theorem. (ii) and (iii) are equivalent because: (ii) $\leftrightarrow G(\leqq a) \in \Sigma_{3}$ by the preceding theorem; $G(\leqq a) \in \Sigma_{3}$ $\leftrightarrow \Sigma_{3}=\Sigma_{3}(a)$ by Theorem 1 and $\Sigma_{3}=\Sigma_{3}(a) \leftrightarrow a^{(2)}=0^{(2)}$ because $\Sigma_{3}=\Sigma_{1}\left(0^{(2)}\right)$ and $\Sigma_{3}(a)=\Sigma_{1}\left(a^{(2)}\right)$. Notice that it is a trivial consequence of Friedberg's work [1] that (i) is equivalent to

(iv) the class of recursively enumerable sets of degree $\leqq a$ is uniformly recursively enumerable without repetitions.

On the other hand it is not possible to add

(v) the elass of recursively enumerable sets of degree $\leqq a$ is uniformly of degree $\leqq a$; since, for example, the recursive sets are certainly not uniformly recursiye even though they are recursively enumerable. We do not know of any recursively enumerable degrees $\boldsymbol{a}<\mathbf{0}^{(1)}$ for which the recursively enumerable sets of degree $\leqq a$ are uniformly of degree $\leqq a$.

\section{REFERENCES}

1. R. M. Friedberg, Three theorems on recursive enumeration, J. Symbolic Logic 23 (1958), 309-316.

2. D. A. Martin, Classes of recursively enumerable sets and degrees of unsolvability, $Z$. Math. Logik Grundlagen Math. 12 (1966), 295-310. 
3. G. E. Sacks, Degrees of unsolvability, Ann. of Math. Studies No. 55, Princeton Univ. Press, Princeton, N. J., 1963.

4. - The recursively enumerable degrees are dense, Ann. of Math. 80 (1964), 300-312.

5. —- A maximal set which is not complete, Michigan Math. J. 11 (1964), 193-205.

6. Y. Suzuki, Enumeration of recursive sets, J. Symbolic Logic 24 (1959), 311.

7. C. E. M. Yates, Three theorems on the degrees of recursively enumerable sets, Duke Math. J. 32 (1965), 461-468.

8. - On the degrees of index sets, Trans. Amer. Math. Soc. 121 (1966), 309-328.

UNIVERSITY OF MANCHESTER,

MANCHESTER, ENGLAND 